\title{
Migración y desarrollo en Chile. Respuestas migratorias a las fases de crecimiento y crisis, 1992-2012
}

\section{Migration and Development in Chile. Migratory responses to economic crisis and growth cycles, 1992-2012}

\author{
Sylvia Soto-Alvarado y Fernando Gil-Alonso
}

\author{
Escuela de Administración Pública de la Universidad Austral de Chile, \\ sede Puerto Montt, Chile \\ Departamento de Geografía, Facultad de Geografía e Historia de la \\ Universidad de Barcelona, Barcelona, España
}

Resumen

Se analiza la interrelación entre los flujos migratorios y las fases de crecimiento —iniciada en los años 90- y decrecimiento económico — crisis de la industria salmonera entre los años 2007 y 2010 - en Chile, tomando la región de Los Lagos como caso de estudio. Los temas abordados son los siguientes: características de la migración interna y su relación con el crecimiento económico regional; los cambios en las tendencias migratorias ante las fases de desarrollo y crisis en la región y, finalmente, la dinámica de la migración por edades y su impacto en las comunas. Para el análisis se utiliza los microdatos de los censos de población y vivienda de los años 1992, 2002 y 2012, este último no validado oficialmente pero que se puede utilizar para fines estadísticos.

Palabras clave: Migración interna, desarrollo, crecimiento económico, crisis, Chile.

Abstract

This paper analyses how migratory flows and economic growth and crisis phases are interrelated in Chile's Los Lagos region, comparing the booming years starting at the beginning of the 1990s with the recessive period due to the salmon industry crisis - years 2007 to 2010. The main subjects treated are the way in which internal migration characteristics and regional economic growth are interlinked, changes in intra-regional migratory trends during the economic growth and crisis periods, and, finally, migration dynamics by age and their impact on Los Lagos' municipalities. 1992, 2002 and 2012 housing and population census microdata are the databases used for the analysis. It should be taken into account that even though the latter census has not been officialy validated, it can nevertheless be used for statistical purposes.

Keywords: Internal migration, development, economic growth, crisis, Chile. 


\section{INTRODUCCIÓN}

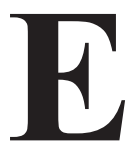

$\mathrm{n}$ los últimos 30 años Chile ha experimentado cambios significativos respecto de las tendencias de desarrollo territorial y, muy ligado con lo anterior, en lo referente a los intercambios de población entre sus comunas, provincias y regiones, lo que se suele calificar como migración interna.

Así, mientras que la región metropolitana ha perdido una parte de su atractivo migratorio, en contraste, algunas regiones históricamente expulsoras de población han cambiado su tendencia mostrándose atractivas como destino migratorio (Rodríguez y González, 2006: 7). Estos cambios se encuentran relacionados con el desarrollo de sectores productivos dedicados principalmente a la exportación, los cuales se han localizado en regiones determinadas del país, incrementando su crecimiento económico y por ello también el atractivo migratorio. Los sectores productivos tradicionales en el rubro exportador son la minería, que se ubica preferentemente en las regiones de Tarapacá, Antofagasta y Atacama; el sector silvoagropecuario, que tiene mayor presencia en las regiones de la zona central del país, O'Higgins y Maule; y el sector de la pesca y la acuicultura, que es nuevo en cuanto al peso relativo en exportaciones y que se debe principalmente al desarrollo de la salmonicultura, fundamentalmente en las regiones de Los Lagos y Aysén (Riffo, 2007), sumándose a partir del 2011 la región de Magallanes.

Si bien el cultivo de salmón se inicia en la década de los 70 en Chile, es en la década de los 80 que la región de Los Lagos emerge como región commodity, productora de salmón. Esto a partir de la apertura de 26 nuevos centros de cultivos entre los años 1983 y 1984, y posteriormente a fines de esa década cuando se introduce el cultivo del salmón atlántico para romper con la estacionalidad de la producción (Amtmann y Blanco, 2001).

Así comienza el crecimiento económico de la región, que entre los años 1990 y 2004 fue en promedio de 5.5 por ciento anual, estando por sobre la media nacional de cinco por ciento (Riffo, 2007). De la misma forma, la tasa de participación regional del PIB nacional se incrementó desde cuatro a 4.62 por ciento entre los años 1996 y 2004. Esto significó un cambio en los flujos migratorios de la región, tradicionalmente emigratoria, convirtiéndose en un territorio con tasas de migración neta positiva de acuerdo a las estadísticas de los censos de 1992 y 2002. 
El año 2007 comienza una crisis de la industria salmonera producto de la aparición del virus ISA, ${ }^{1}$ que terminó con producciones completas de salmones, con el cierre de empresas del sector y con despidos laborales masivos, que habría causado cerca de 15 mil desempleados. ${ }^{2}$

La crisis del salmón o del virus ISA en la región impactó en el producto interno bruto regional de tal forma que el año 2009 la variación anual en relación al año 2008 fue de -6.4 por ciento. La economía regional comienza a recuperarse lentamente a partir del 2010, y en el año 2011 se observa una variación de 11.5 por ciento en el PIB en relación con 2010, mostrándose un gran impulso económico.

Los centros de cultivo acuícola se encuentran en algunas comunas específicas de la región, mientras que las gerencias o niveles decisionales más altos de la industría salmonera se encuentran de preferencia en la Ciudad de Puerto Montt, capital de la región de Los Lagos, y en la ciudad de Santiago, capital de Chile. Por lo tanto, el atractivo migratorio de la región no es homogéneo en el interior de ésta, generando corrientes migratorias internas que profundizan las diferencias de recurso humano disponible en los territorios (Rodríguez, 2008).

Rodríguez y Busso (2009) señalan que la migración interna incide de manera directa sobre las brechas territoriales, causando despoblamiento en comunas pequeñas con poca actividad económica productiva y robusteciendo las comunas que son el motor de desarrollo regional, exacerbando por lo tanto las desigualdades y creando una clara heterogeneidad etaria entre las regiones, o entre las comunas de una región, en función de si son inmigratorias o emigratorias. Estas diferencias impactan directamente en las posibilidades de desarrollo de los municipios pequeños y rurales de la región, dado que quedan despoblados de hombres y mujeres en edad productiva laboralmente.

Analizar la relación entre desarrollo económico regional y migraciones internas en las últimas décadas es el objetivo de este artículo, que toma la región de Los Lagos como caso de estudio por ser el prototipo de región commodity y por haber experimentado, en un corto lapso de tiempo, fases de fuerte crecimiento económico y de profunda crisis, lo que nos permitirá

\footnotetext{
${ }^{1}$ Virus ISA se le denomina a la Anemia Infecciosa del Salmón, una enfermedad altamente contagiosa que ataca preferentemente al salmón del Atlántico. El brote de la enfermedad se genera por la transmisión de una población local o por importación del exterior. No existe un acuerdo sobre la vía por la cual llegó el virus ISA al país. El ISA se propagó en tres regiones del país en las que se encuentran la mayor cantidad de centros de cultivo de salmón.

${ }^{2}$ No se cuenta con una cifra oficial sobre la cantidad de despidos producto de la crisis del salmón, sin embargo, distintas fuentes se acercan a esta cifra, que además es mencionada en el seminario universitario impartido por Gillet y Olate (2010), "La crisis del salmón y el desempleo en la décima región".
} 
testar cómo responden los flujos migratorios a los cambios de coyuntura económica, y cómo estos movimientos de población impactan a su vez en el desarrollo de los territorios de origen y destino. Para ello se analizará también la dinámica de la migración por edades y su impacto en las comunas, lo que nos permitirá sugerir finalmente algunas medidas políticas que podrían atenuar los impactos negativos.

\section{Marco analítico}

\section{Migración interna y desarrollo}

Ciertamente las desigualdades existentes entre territorios es un desencadenante de los flujos migratorios (Rodríguez, 2008), pero así también la migración aumenta las desigualdades estructurales entre territorios, tanto a nivel rural-urbano, como entre regiones. (Todaro, 1980). Es habitual que migre la población con mayor calificación y en edad laboral, aumentando así los desequilibrios territoriales. Un papel importante en la regulación de los flujos migratorios internos son las políticas sociales y económicas aplicadas en los territorios, que pueden favorecer o no el desarrollo endógeno. Sin embargo, en Chile hay escasas políticas locales de regulación para el crecimiento y desarrollo del sector económico industrial.

En cuanto a los factores que mueven a las persones a migrar, la teoria clásica postula que el estímulo primario para la migración es la racionalidad económica, expresada en la diferencia de empleo y salarios entre un territorio y otro. Y que finalmente, es la expectativa de obtener mayores ingresos económicos más que la diferencia real de ingresos entre un territorio y otro, lo que impulsa a las persones a migrar (Todaro, 1980). No se puede dejar de mencionar que la decisión de migrar no obedece solamente a motivos económicos, sino que existen otros factores, de tipo social y cultural, que hacen más probable la migración, como la existencia de redes interpersonales que conectan a los futuros migrantes con otros migrantes en el lugar de destino y los soportes institucionales de apoyo a la migración (Massey 1990; Massey et al., 1993).

Rodríguez (2011: 52) señala que en América Latina hay diversos estudios que sostienen que, si bien la migración interna es un proceso sobre el cual no se puede hacer generalizaciones, pues cada caso presenta sus propias particularidades y por ello hay que analizarlo en función del contexto específico de la región o del país, sí es posible establecer en términos macro una relación entre migración y proceso de desarrollo. Estudios de la CEPAL han pasado desde una mirada funcionalista hasta una mirada es- 
tructural, proponiendo en las publicaciones del último periodo un "marco general de los determinantes y consecuencias de la migración" (Rodríguez y Busso, 2009: 27). En este planteamiento

se reconocen elementos jerárquicos de determinación y afectación, marcando así la distinción central entre corrientes que se mueven de lugares de destino a lugares de origen y decisiones individuales de migrar (Rodríguez y Busso 2009: 41).

A nivel macro, se encuentra como determinante el nivel y estilo de la dinámica de desarrollo, que se refiere a aquellos factores involucrados en el modelo desarrollo y que inciden en las migraciones, como el nivel de apertura a las exportaciones, el tipo de productos exportados, los distintos niveles de productividad de los territorios, entre otros.

En un segundo nivel o nivel meso, se encuentran los factores económicos, sociales y culturales que influyen en la decisión individual de migrar, que a su vez están determinados por el estilo y la dinámica de desarrollo existente. Así, encontramos entre los factores económicos las diferencias de ingreso entre un territorio y otro, las posibilidades de empleo y los costos de traslado que puedan estar asociados. En los factores sociales y culturales, se encuentran las diferencias territoriales de calidad de vida, de educación, de acceso a oportunidades que permitan un mejor nivel de vida. Tanto el estilo de desarrollo (nivel macro) como los factores económicos, sociales y culturales que resulten de este (nivel meso), se encuentran influenciados por lo que haga o deje de hacer el Estado. Esto último, a diferencia de los análisis de migraciones en países europeos, es de gran importancia en América Latina, debido a las políticas públicas de desarrollo y concentración/desconcentración regional.

Finalmente, a un nivel micro están los determinantes psicosociales y de oportunidad en la decisión individual —o de una familia - de migrar. En ellos se contempla factores de tipo personal que contribuyen en la toma de decisiones como la edad, sexo, tipo de familia, nivel de educación, predisposición al cambio, entre otros.

\section{Contexto y factores que determinan la migración interna en Chile}

Tomando como base lo explicado en las páginas anteriores, se considera que la migración interna en Chile está determinada por diversos factores; de ellos, cuatro serían los más importantes (Rodríguez y González, 2004). El factor principal es el atractivo que tienen las regiones, tanto de tipo económico como de tipo sociocultural, y de oferta de mejoramiento de la ca- 
lidad de vida. Un segundo factor es la conectividad, que ha tomado fuerza en el último tiempo principalmente en ámbitos metropolitanos, puesto que se considera el hecho de la reducción de costos y tiempos en los desplazamientos de corta escala. El tercer factor que se menciona es la historia, entendiendo que a través de ella se produce un "efecto de inercia sobre los flujos y las decisiones migratorias". Además, se incluyen en este factor las redes sociales, puesto que éstas consolidan un flujo de emigrantes hacia un lugar determinado en función de los primeros inmigrantes que llegan a un destino y que, después, producto de los canales de información y la existencia de esta red, se mantiene estable. Un último factor relevante, en el caso de Latinoamérica, como en el particular de Chile, son las políticas públicas, que influyen directa e indirectamente en los flujos migratorios debido a las decisiones que tienen impacto en determinados territorios como, por ejemplo, la asignación de recursos públicos o la concesión de incentivos salariales o tributarios.

Los factores mencionados (nivel meso), que influyen en las decisiones migratorias de individuos y familias (nivel micro), se encuentran y operan en un contexto más amplio (nivel macro) determinado por el modelo económico, la dinámica de producción nacional/regional, la ideología y los aspectos socioculturales que definen los márgenes de posibilidades (Rodríguez, 2004).

Para explicar las consecuencias espaciales de este contexto, en Chile se han desarrollado dos hipótesis que intentan explicar la evolución de este país desde la perspectiva económica y de desarrollo, y que a su vez $-\mathrm{y}$ eso es lo que nos interesa en este artículo - tienen implicaciones de cara a explicar la variación de los flujos migratorios internos.

Desde la mirada económica tradicional, Daher (1994) plantea que Chile está viviendo un proceso desconcentrador, en el que la Región Metropolitana ha tenido, en los últimos 20 años, un crecimiento económico a un ritmo más bajo que el promedio nacional, y ya no es el atractivo principal del potencial migratorio del país (Daher, 1994: 64-65). Por el contrario, han aparecido regiones históricamente postergadas en cuanto a su capacidad de producción y crecimiento económico, como ganadoras. Este planteamiento se fundamenta en que el perfil exportador del país y las ventajas comparativas existentes en algunas actividades productivas han consolidado la posición de algunas regiones que actualmente presentan rubros dinámicos, principalmente en la exportación de productos primarios, más conocidas como "regiones commodities". En consecuencia, Daher concluye que: "el proceso de apertura económica y el desarrollo exportador han detonado un 
proceso de ajuste territorial de carácter estructural del que emergen nuevas regiones transables de exportación y domésticas" (Daher, 1994: 82), debilitando la primacía de las regiones donde históricamente se ha concentrado la economía del país, la metropolitana y la de Valparaíso.

Uno de los principales expositores de la segunda tesis, aplicada a Chile, es De Mattos (1995) quien señala que la nueva estrategia macroeconómica descentralizada y de libre mercado implantada en Chile desde mediados de 1970 permitió el desarrollo de cuatro cadenas productivas principales, orientadas al mercado exterior: hortofrutícola, minera, pesquera y forestal, lo que intensificó el dinamismo de las regiones donde estas se encuentran. Esta evolución es la que habría llevado a algunos autores a defender la existencia de una mayor descentralización productiva en el territorio chileno. Sin embargo, si bien ha aumentado el desarrollo de estas regiones y de sus ciudades intermedias, el capital ha continuado concentrándose en grandes grupos económicos que, al mismo tiempo, han centralizado y concentrado las funciones de más alta jerarquía en el territorio metropolitano.

De Mattos analiza el debate sobre divergencia o convergencia y afirma que, aunque la discusión original es sobre la convergencia económica de países, este debate se ha trasladado al nivel nacional para ver la convergencia entre regiones. Él plantea que la tendencia a la convergencia no constituye un rasgo inherente a la nueva dinámica económica desencadenada bajo los efectos de la reestructuración y la globalización (De Mattos, 1999; 2000). De hecho, el reconocimiento de la presencia de externalidades y rendimientos crecientes otorga fundamento a la hipótesis de que la localización de la cabeza de los principales procesos productivos - así como también de la parte más moderna e innovadora de la industria y del terciario avanzado - se realiza predominantemente en algunos centros urbanos desde donde se ejerce el comando de un elevado número de territorios periféricos que, dado su limitado potencial endógeno, carecen de la capacidad necesaria para poder revertir las desigualdades que les afectan debido a la acumulación de capital a escala internacional, nacional y regional (De Mattos, 2000: 39).

En el caso de Chile, se han realizado análisis para evaluar la hipótesis de convergencia, sin embargo, no se ha llegado a resultados que la aprueben o rechacen completamente (Rodríguez y González, 2006). Estos autores señalan que estudios recientes muestran que el proceso de convergencia ha sido más bien errático en Chile y que en los últimos años se tendería a la divergencia económica entre las regiones del país. Pero lo mismo se podría decir de los procesos de desarrollo en el interior de las regiones, de ahí la 
pertinencia de un estudio como el presente, que para verificar las hipótesis de convergencia o divergencia analiza las migraciones internas en la región de Los Lagos y su conexión con las fases de coyuntura económica, proponiendo finalmente algunas medidas políticas que podrían favorecer el desarrollo de las comunas más deprimidas.

\section{Metodología y fuentes de datos}

Para el análisis se utilizaron los microdatos de los censos de población y vivienda de Chile de los años 1992, 2002 y 2012. Las preguntas básicas que se consideraron para el análisis son dos: el lugar de residencia habitual al momento de efectuar la encuesta censal para determinar la población de derecho en las comunas, y lugar de residencia cinco años atrás en el momento que se realiza el censo, con el fin de determinar el último movimiento de la población.

A partir de dichos datos, los indicadores utilizados para el análisis demográfico son los siguientes:

- $\quad$ Migración Neta o Saldo Migratorio = Inmigrantes - Emigrantes

- Tasa Bruta de Inmigración $(\mathrm{TINM})=((\mathrm{I} /$ núm. de años $) /$ Pobl. total media) $* 1000$

- Tasa Bruta de Emigración $(T E M)=((E / n u ́ m$. de años $) /$ Pobl. total media)*1000

- $\quad$ Tasa de Migración Neta $(\mathrm{TMN})=\mathrm{TINM}-\mathrm{TEM}$

En el caso de los indicadores para medir migración, en el denominador de las tasas se utilizó como población total, en ambos censos, la población de derecho mayor de cinco años, concretamente la media entre población inicial y final, es decir, separadas por cinco años.

\section{Consideraciones sobre el censo de población y vivienda del año 2012}

El censo del año 2012 no ha sido validado por el gobierno de Chile para la estimación y proyecciones de población ni para la toma de decisiones en el ámbito de políticas públicas. Sin embargo, los microdatos han sido puestos a disposición de los investigadores para la realización de estudios, teniendo la debida cautela en el uso de la información.

Uno de los principales problemas del censo 2012 respecto de las variables demográficas es el porcentaje de omisión, que según los cálculos del INE sería de 9.6 por ciento, superando ampliamente la omisión de censos anteriores. Para la región de Los Lagos el porcentaje de omisión calculado 
es de 9.7 por ciento. ${ }^{3}$ Sobre la migración interna reciente, el documento de la auditoria a la base de datos censal señala que hay una "posible subestimación de la migración interna" que dificultaria obtener los stocks de migración interna.

En la ronda de censos del año 2000 en América Latina, la mayor omisión censal fue de 7.7 por ciento y el promedio de omisión de los paises fue de 3.2 por ciento (Chackiel, 2009), por lo que la omisión del censo 2012 de Chile está muy por encima de lo esperado. Sin embargo, los datos obtenidos en los procesos censales no están exentos de error, pero éstos pueden ser rehabilitados o rescatados para su uso. Un elemento clave para el uso y credibilidad de los datos censales es la transparencia del proceso, por ello, la disponibilidad de la auditoría de la base de datos censal es fundamental para el uso de los datos.

Pese a los errores encontrados a la información censal de 2012, ésta sigue siendo la base de datos más actualizada sobre población en Chile que puede dar cuenta de los procesos demográficos de los últimos diez años en el país.

\section{Consideraciones sobre la comuna de Chaitén}

La comuna de Chaitén es la capital de la provincia de Palena, una de las cuatro provincias de la Región de Los Lagos. Su población en el año 2002, según cifras del INE, era de 7,182 personas, entre población urbana y rural.

El 2 de mayo de año 2008, de forma imprevista, hizo erupción el volcán Chaitén ubicado a aproximadamente diez kilometros de la ciudad de Chaitén, lo que obligó a realizar una evacuación rápida de la población urbana y rural de los alrededores, cifra que se estima en 4,100 evacuados. Sin posibilidad de estimar la duración de la actividad del volcán y estando la ciudad ubicada en una zona de riesgo, la población urbana fue desplazada (aproximadamente cuatro mil personas) debiendo relocalizarse en otras ciudades de las provincias de Palena, de Chiloé y de Llanquihue.

Esta migración forzada producto del desastre natural se observa en las cifras del censo 2012, sin embargo, como no es parte del objectivo de este estudio, no se han relevado en el análisis de los resultados.

\footnotetext{
${ }^{3}$ De acuerdo a lo que señala la Auditoría Técnica a la base de datos del levantamiento censal año 2012, realizada por el Instituto Nacional de Estadísticas de Chile el año 2014.
} 


\section{RESULTADOS: DINÁMICA MIGRATORIA INTERNA EN LA Región DE LOS LAGOS}

Las estadísticas del INE señalan que la región de Los Lagos fue, hasta el censo de 1992, una región expulsora de población teniendo una TMN interregional negativa que sólo se revirtió a partir del año 2002. No obstante, se ha de tener en cuenta que hasta el año 2007 la región de Los Lagos estaba constituida por cinco provincias - Valdivia, Osorno, Llanquihue, Chiloé y Palena- y que posteriormente la provincia de Valdivia (fuertemente emigratoria) se separó de la región de Los Lagos y se constituyó como la región de Los Ríos. Para que los resultados del presente artículo sean homogéneos, se han incluido, en los tres periodos analizados, solamente las comunas pertenecientes a cuatro actuales provincias de la región. Tomando, por lo tanto, la actual división político administrativa de la región, el análisis de las migraciones interregionales demuestra que ya en el censo del año 1992 la región de Los Lagos tenía una tasa de migración neta positiva (1.34 por ciento, véase la Tabla 1). Estos datos demuestran que ya en 1992 se había registrado un cambio en la tendencia migratoria histórica de Los Lagos, perfilándose así, como una región atractora de población.

Este cambio en el patrón migratorio coincide con el auge de la industria salmonera, que tuvo su mayor crecimiento entre los años 1990 y 2007, y que generó alrededor de 50 mil puestos de trabajo en forma directa e indirecta en el país ${ }^{4}$. Estos últimos relacionados con la prestación de servicios y equipamientos para el funcionamiento de la industria.

El censo del año 2012 muestra que en los últimos cinco años la región volvió a tener un saldo migratorio negativo, con una tasa de migración neta de -0.47 por ciento. Sin duda, la crisis de la industria salmonera y la pérdida de las fuentes de trabajo impactó en los flujos de población en la región (ver Tabla 1).

La escasa diversificación de los rubros productivos en la región, y la disminución del trabajo agrícola tradicional, no fue capaz de absorber el desempleo que se produjo entre los años 2009 y 2010 producto de la crisis del salmón. El gobierno regional y los gobiernos locales implementaron medidas de mitigación del desempleo que lograron disminuir la cesantía ligada a la crisis del sector, orientada principalmente a los trabajadores de baja calificación.

\footnotetext{
${ }^{4}$ De acuerdo a informe Radiografía a la Industria del Salmón en Chile bajo la mirada de e standares de RSE en http://www.mapeo-rse.info/sites/default/files/Radiografia a la industria del_salmon.pdf, consultado el 25 de abril del 2016.
} 
Tabla 1: Tasas de inmigración, emigración y de migración neta, por periodos, Región de los Lagos. censos 1992-2012

\begin{tabular}{lrrr}
\hline $\begin{array}{l}\text { Región de } \\
\text { Los Lagos }\end{array}$ & $\begin{array}{r}\text { Tasa de } \\
\text { inmigración }\end{array}$ & $\begin{array}{r}\text { Tasa de } \\
\text { emigración }\end{array}$ & $\begin{array}{r}\text { Tasa de migración } \\
\text { neta }(\%)\end{array}$ \\
\hline $1987-1992$ & 14.54 & 13.19 & 1.34 \\
$1997-2002$ & 16.59 & 12.06 & 4.54 \\
$2007-2012$ & 11.82 & 12.29 & -0.47 \\
\hline Fuente: elaboración propia con microdatos de los censos 1992,2002 y 2012 del INE-Chile
\end{tabular}

La emigración hacia otras regiones en el último periodo se relaciona entonces con migración de retorno y con la búsqueda de nuevas oportunidades de la población con mayor calificación.

\section{Patrones geográficos de las migraciones internas}

La dinámica migratoria interna en la región de Los Lagos tiene un componente importante de éxodo rural hacia las zonas urbanas, ya que en la región aún hay 30.37 por ciento de población rural, a diferencia de la población rural nacional que es 12.79 por ciento. Esta dinámica puede explicarse en términos generales a través de las clásicas teorías push - pull, pues es en los centros más poblados donde se concentran las oportunidades laborales y también los centros de educación superior.

El porcentaje de urbanización en la región aumenta en las comunas con ciudades intermedias de la región, donde la población urbana llega aproximadamente a 85 por ciento o más. Si no hay cambios en las políticas públicas y en la localización de inversiones privadas, la tendencia es que continúe el progresivo despoblamiento de las comunas de carácter rural.

El 39.9 por ciento del flujo de inmigrantes internos en el periodo 198792 se concentraba en las comunas capitales de provincia, donde también vivía la mitad de la población de la región. Sin embargo, la progresiva atracción de las áreas urbanes hizo que, en los periodos 1997-2002 y 2007-2012, esta proporción aumentara hasta llegar a 54.21 por ciento. La mitad de esta cifra se asienta en la comuna de Puerto Montt, capital regional (ver Tabla 2).

Particular es la situación en la provincia de Palena, cuyas comunas - de escasa población y baja densidad demográfica - se encuentran aisladas del resto de la región y del país, por ello los flujos de inmigrantes que reciben son reducidos. 


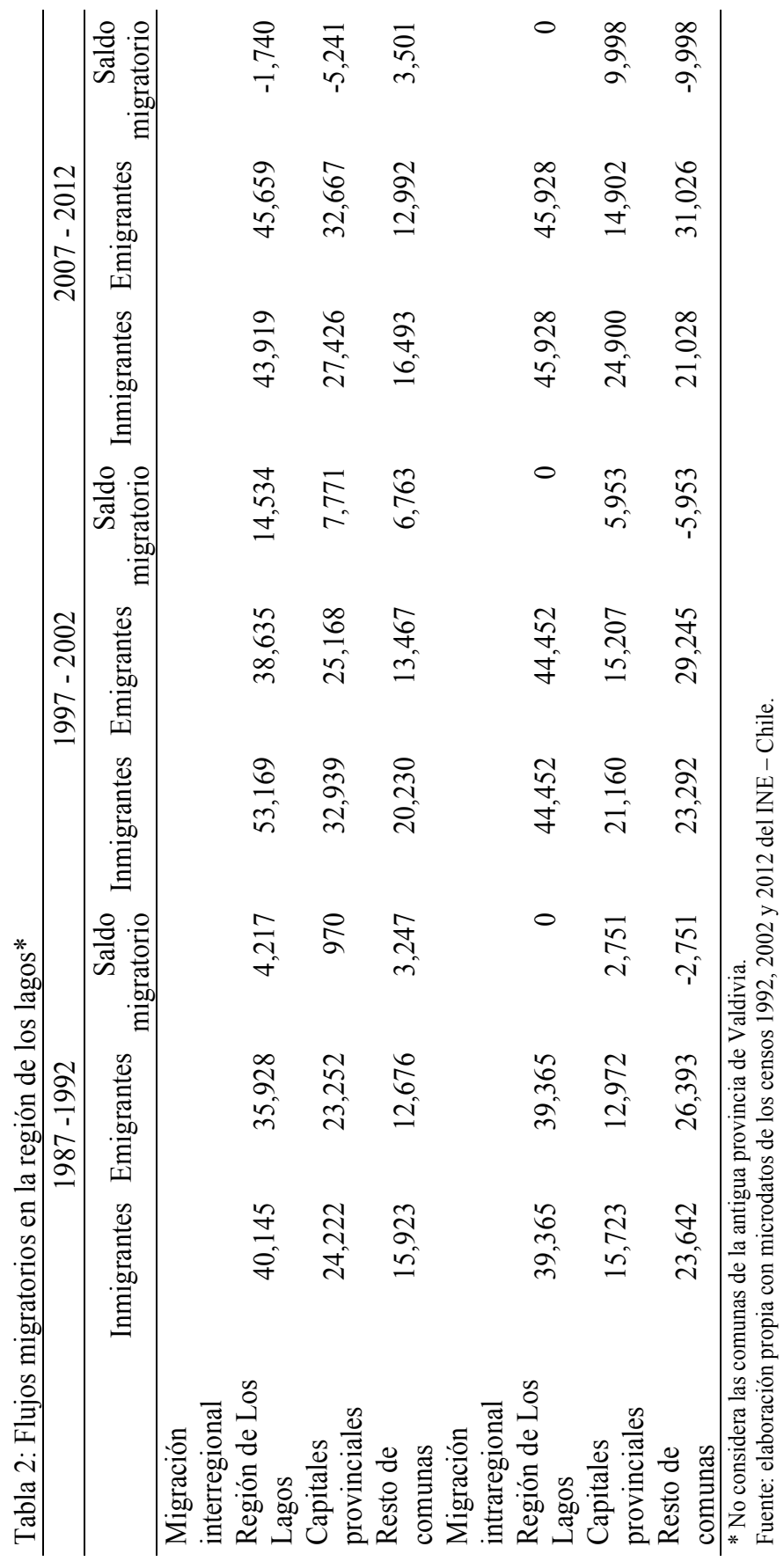


El auge de la industria acuícola y su posterior crisis impacta directamente en los flujos migratorios de la región. La intensidad de la inmigración aumentó durante los años de mayor crecimiento económico, sobre todo en las comunas de desarrollo acuícola, para mantenerse en toda la región en el periodo 2007-2012.

Este ciclo se observa también en el atractivo migratorio que supone la región para migrantes de otras regiones del país, puesto que, de acuerdo a los datos del censo de 2002, 52.5 por ciento de la inmigración en la región provino de otras regiones del país.

La Figura 1 muestra que los flujos de inmigrantes internos son más intrarregionales en todas las comunas que los flujos de inmigrantes interregionales. Los flujos de inmigrantes interregionales son más selectivos en cuanto a los destinos en la región. Las tasas de inmigración interregional más altas se encuentran en las comunas que son capitales provinciales, comunas con desarrollo turístico y comunas con desarrollo ligado a la acuicultura.

En el periodo 1987-1992, las comunas con tasas de inmigración más alta, además de las capitales provinciales, son comunas que bordean el lago Llanquihue y algunas de las provincias de Chiloé y Palena, especialmente la comuna de Dalcahue y la de Quellón, que mantienen las altas tasas de inmigración en el periodo de 1997-2002. Estas comunas han tenido en las décadas del 90 y 2000 un fuerte auge económico y demográfico ligado a la producción de la industria acuícola, debido a la instalación de centros de cultivos de salmones y mitílidos de empresas principalmente extranjeras.

En el periodo 2007-2012 las comunas que habían presentado tasas más altas de inmigración disminuyen drásticamente debido al cierre de centros de cultivo de salmones y la disminución de las fuentes laborales. De la misma forma disminuye la inmigración hacia la capital regional, Puerto Montt, donde se encuentran centros de proceso de materias primas y las gerencias de las empresas salmoneras. Por último, ante la erupción del volcán Chaitén el año 2008, parte de la población evacuada se asentó posteriormente en las comunas de Palena y Futaleufú de la misma provincia, por ello presentan las tasas de inmigración más altas de la región en el último censo de población.

La emigración interregional es menos intensa que la emigración intrarregional. Las tasas más altas de emigración interregional se encuentran en las capitales provinciales, Puerto Montt, Osorno y Castro, en cambio tasas más altas de emigración intrarregional se observan en las comunas rurales, principalmente las de desarrollo agropecuario. 
Figura 1: Tasas de inmigración interregional (izquierda) e intrarregional (derecha) comunas de la región de los lagos, según censos $1992-2002-2012$

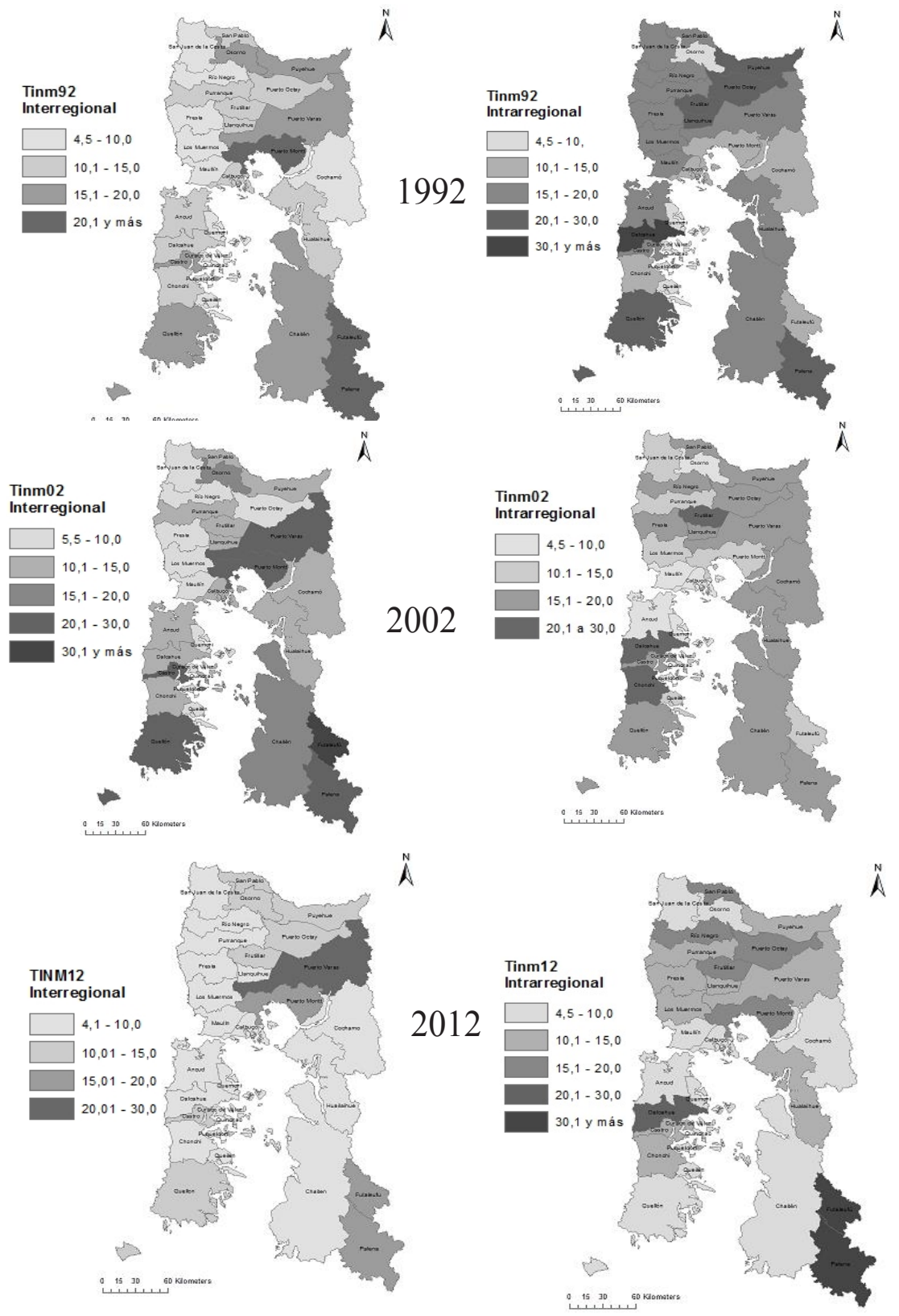

Fuente: elaboración propia con microdatos de los censos 1992, 2002 y 2012 del INE - Chile. 
En los tres periodos analizados las tasas de emigración son más bajas en la isla de Chiloé, siendo coherente con el desarrollo acuícola de las comunas de la provincia.

Las tasas de emigración en la región (Figura 2) fueron más altos en los periodos 1987-1992 y 1997-2002, son décadas marcadas por la migración campo-ciudad y el movimiento hacia comunas con mayores opciones de trabajo. Las comunas más emigratorias en ambos periodos son las que tienen una economía fundamentalmente agraria, al Noroeste y al Sureste de la región. Para el periodo 2007-2012, sin embargo, se observa un descenso de las tasas de emigración en las comunas de la región, principalmente en las comunas rurales del borde costero de producción agropecuaria. Una de las razones de este descenso es la inversión pública en infraestructura y fomento productivo que se ha realizado en los últimos 20 años en las zonas rurales, mejorando la conectividad y las posibilidades de desarrollo. Junto con ello, la "desaparición" durante los años 2008-2010 de la producción de salmón dejó en condiciones de alta vulnerabilidad y pobreza a las comunas que habían basado su crecimiento en este sector. Así, la crisis económica disminuyó el movimiento de población en el interior de la región, principalmente las migraciones entre comunas pequeñas con mayor desarrollo económico vinculado a la producción acuícola, como son las comunas Quellón, Dalcahue, Cochamó, Hualaihue, entre otras.

La excepción en la disminución de los flujos emigratorios la constituye la comuna de Chaitén producto de la erupción del volcán Chaitén, y Puerto Varas, una comuna lacustre de no más de 35 mil habitantes que limita con Puerto Montt y que, el año 2012, vio como una parte de su población fue transferida a la comuna de Puerto Montt, al anexarse a esta comuna la localidad de Alerce Norte. Esto ha incidido a su vez, en las tasas de migración neta de las dos comunas.

En los tres periodos analizados la migración neta intrarregional positiva se encuentra focalizada en la capital regional, Puerto Montt, su comuna aledaña de Puerto Varas y las comunas de la Provincia de Chiloé, mientras que la mayoría de las comunas rurales presentan unas tasas de migración neta intrarregional negativa (Figura 3). Sin embargo, en el periodo 20072012 las tasas de migración neta intrarregional disminuyeron en toda la región, dando cuenta de la crisis económica de la industria del salmón. Únicamente la capital regional, Puerto Montt, las comunas que recibieron inmigrantes debido a la erupción del volcán Chaitén, y las comunas de Chiloé donde se ha desarrolla la industria de mitílidos, muestran fuertes tasas netas positivas. 
Figura 2: Tasas de emigración interregional e intrarregional de las comunas de la región de los lagos,

$1992-2002-2012$
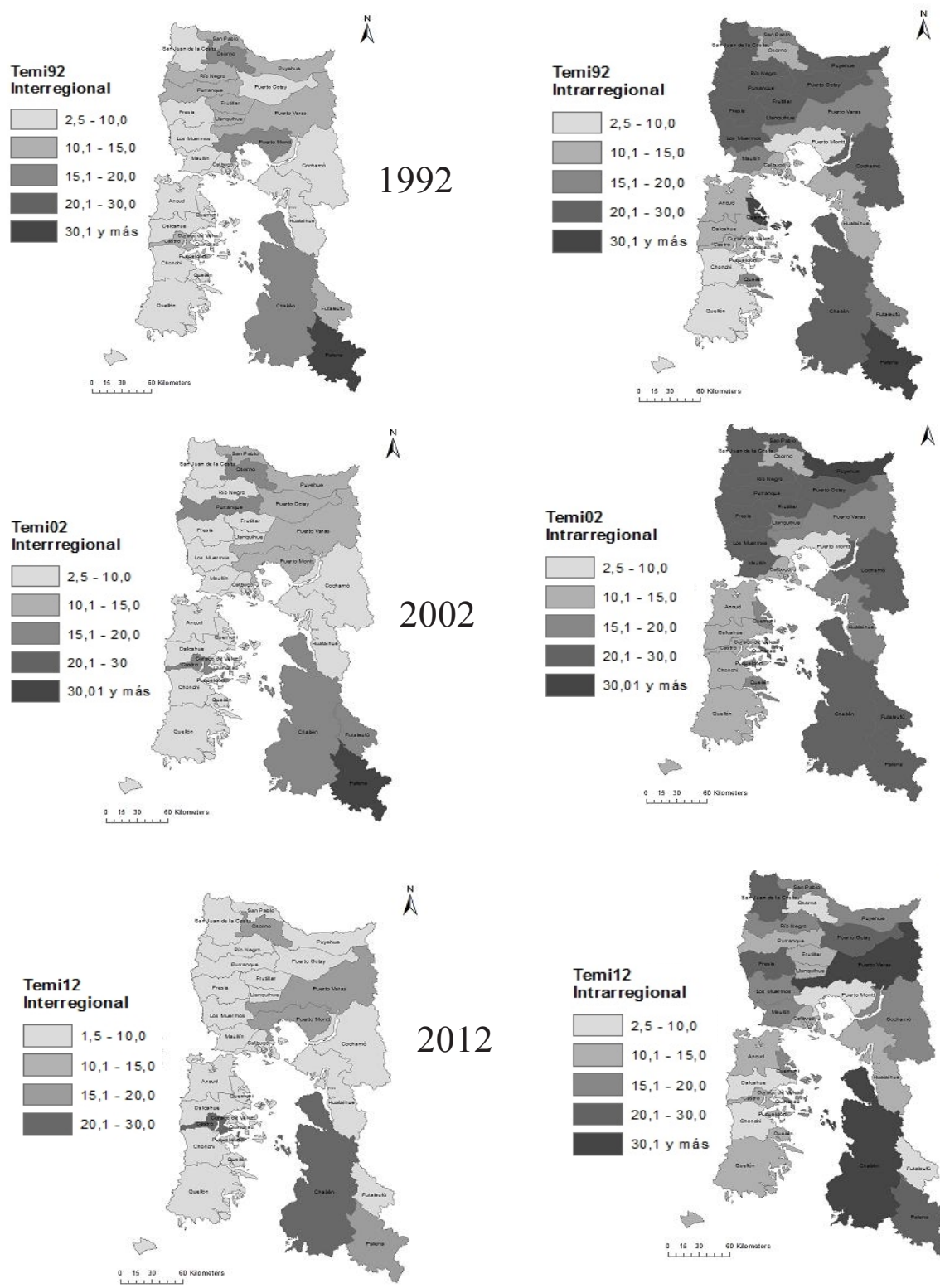

Fuente: elaboración propia con microdatos de los censos 1992, 2002 y 2012 del INE - Chile. 
Migración y desarroollo en Chile. Respuestas migratorias a las fases de crecimiento y crisis ... / S. SOTO y F. GIL

Figura 3: Tasas de migración neta interregional e intrarregional, censos 1992, 2002 y 2012

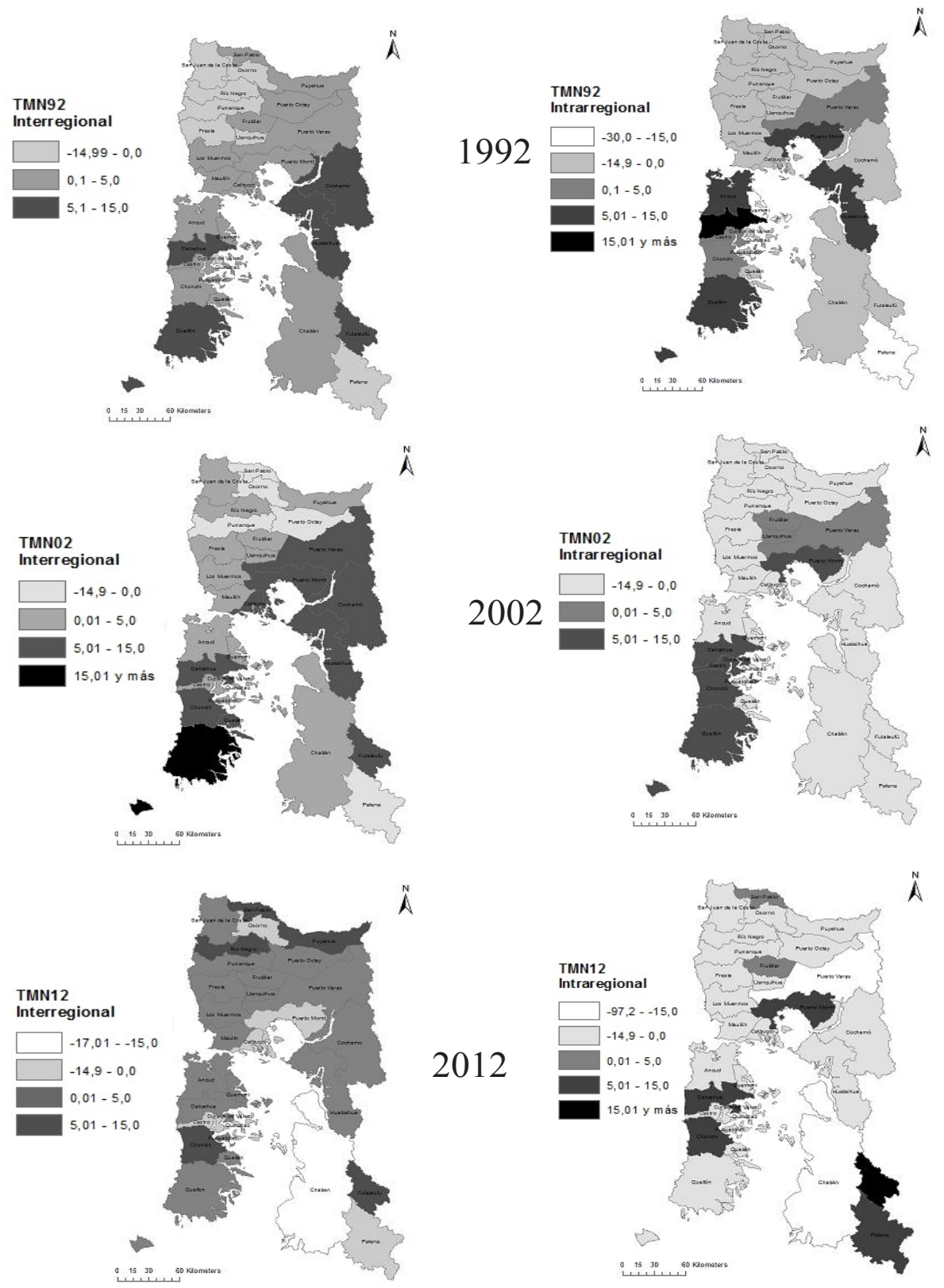

Fuente: elaboración propia con microdatos de los censos 1992, 2002 y 2012 del INE - Chile. 
Las tasas de migración neta (TMN) interregional son más altas en las capitales de región y provincias y en las comunas de desarrollo acuícola, al igual que la migración neta intrarregional, pero mayor cantidad de comunas de la región tiene TMN interregionales positivas, debido a que, en general, la región de Los Lagos es un territorio atractivo para inmigrantes de otras regiones. Además, los flujos migratorios desde las comunas rurales son de la región se dirigen preponderantemente hacia las ciudades intermedias de la propia región y no hacia otras regiones.

La crisis económica de la región de Los Lagos transformó la dinámica migratoria de la región. Así, comunas que ganaban población se han transformado en expulsoras de población en los últimos años mientras que comunas que sistemáticamente han tenido TMN negativas — como algunas del Noroeste de la región - han pasado a ser receptoras de población proveniente de otras regiones. Sin embargo, en cuanto a la migración interna, continúan TMN negativas en la mayoría de las comunas, concentrándose los flujos depoblación en la comuna de Puerto Montt, Dalcahue y Chonchi. Por su parte las comunas Futaleufú y Palena aparecen con TMN producto de la relocalización de la población desplazada de Chaitén.

En tanto, las comunas de Puyehue, San Pablo y Río Negro, que presentaron TMN interregionales negativas en los censos del 1992 y 2002, para el censo de 2012 tienen TMN positivas, dando también las primeras luces de una transición en la dinámica migratoria en la región y en el país. Ello podría significar que están comenzando a disminuir las brechas de desarrollo entre las comunas de la región, o ser un efecto puramente coyuntural como consecuencia de la crisis de la industria salmonera.

De las capitales provinciales, solamente Puerto Montt, como capital regional y provincial mantiene una TMN intrarregional positiva y, en cambio, las comunas de Osorno y Castro son expulsoras de población, lo cual se podría interpretar como que se ha ido acentuando el centralismo político - administrativo y económico de la región.

En resumen, el crecimiento económico de la región a través de la industria acuícola contribuyó a polarizar la dinámica migratoria de las comunas de la región. Mientras las comunas rezagadas económicamente se volvieron más expulsoras de población, las comunas que desarrollaban actividades de la salmonicultura tenían altos flujos de inmigrantes. Con la crisis económica hubo una ralentización de los flujos migratorios en toda la región y una cierta modificación de la dirección de los flujos, mostrando la fragilidad propia de una región commodity que basa el crecimiento económico en un único eje productivo. 


\section{Pautas de la migración en la región por edad}

Los patrones migratorios según la edad de los migrantes muestran una gran regularidad, pues los mayores flujos de inmigrantes se concentran entre los 20 y 40 años (Figura 4). Esta es una constante para casi todas las comunas de la región, existiendo solamente algunas variaciones, entre las cuales destaca la comuna de Puerto Varas, donde la tasa de inmigración más alta se encuentra entre los 30 y 34 años, a diferencia de las demás comunas en las que se produce antes de los 30 años. Puerto Varas también tiene tasas de inmigración más altas que los demás centros urbanos, en todas las edades y con un leve aumento a los 60 años y después de los 70 .

Las restantes comunas ubicadas en la cuenca del Lago Llanquihue también incrementan levemente sus tasas de inmigración a partir de los 70 años. Esto se debe a que dichas comunas, situadas en un entorno ambiental privilegiado, atraen migraciones por causas residenciales tanto de persones mayores como de parejas jóvenes con hijos pequeños (este es especialmente el caso de Puerto Varas, situado cerca de la capital Puerto Montt). Esta capacidad de atracción se aprecia también en las demás comunas que son centros urbanos, pero con valores más bajos.

Por su parte, la emigración de las comunas rurales de la región se produce en edades más tempranas que en las comunas que son centros urbanos (Figura 5). Los flujos más altos en las comunas rurales son entre los 15 y 19 años, cuando los jóvenes que continúan estudios secundarios deben hacerlo en las ciudades con mayor oferta educativa; en cambio las tasas más altas de emigración en los centros urbanos de la región se encuentran a la edad de 20 a 24 años, donde la movilidad se produce por motivos de trabajo y/o estudios universitarios.

Las tasas de emigración de las comunas de cordillera — como Palena y Futaleufu - son muy altas en comparación con las tasas de las demás comunes. Esto se debe a la escasa oferta educativa que existe en estos territorios producto de la lejanía y aislamiento, a las cuales se accede en forma aérea o terrestre a través de Argentina o de una carretera bimodal, que combina transporte terrestre y marítimo, teniendo los viajes aproximadamente nueve horas de duración. Por ello, una porción importante de los jóvenes de 14 años migra para continuar sus estudios en alguna de las capitales provinciales. Por otro lado, es importante mencionar que la oferta educativa de enseñanza superior se encuentra concentrada en las comunas de Osorno y de Puerto Montt, lo que obliga a los jóvenes de otras comunas a migrar si desean continuar estudiando. 

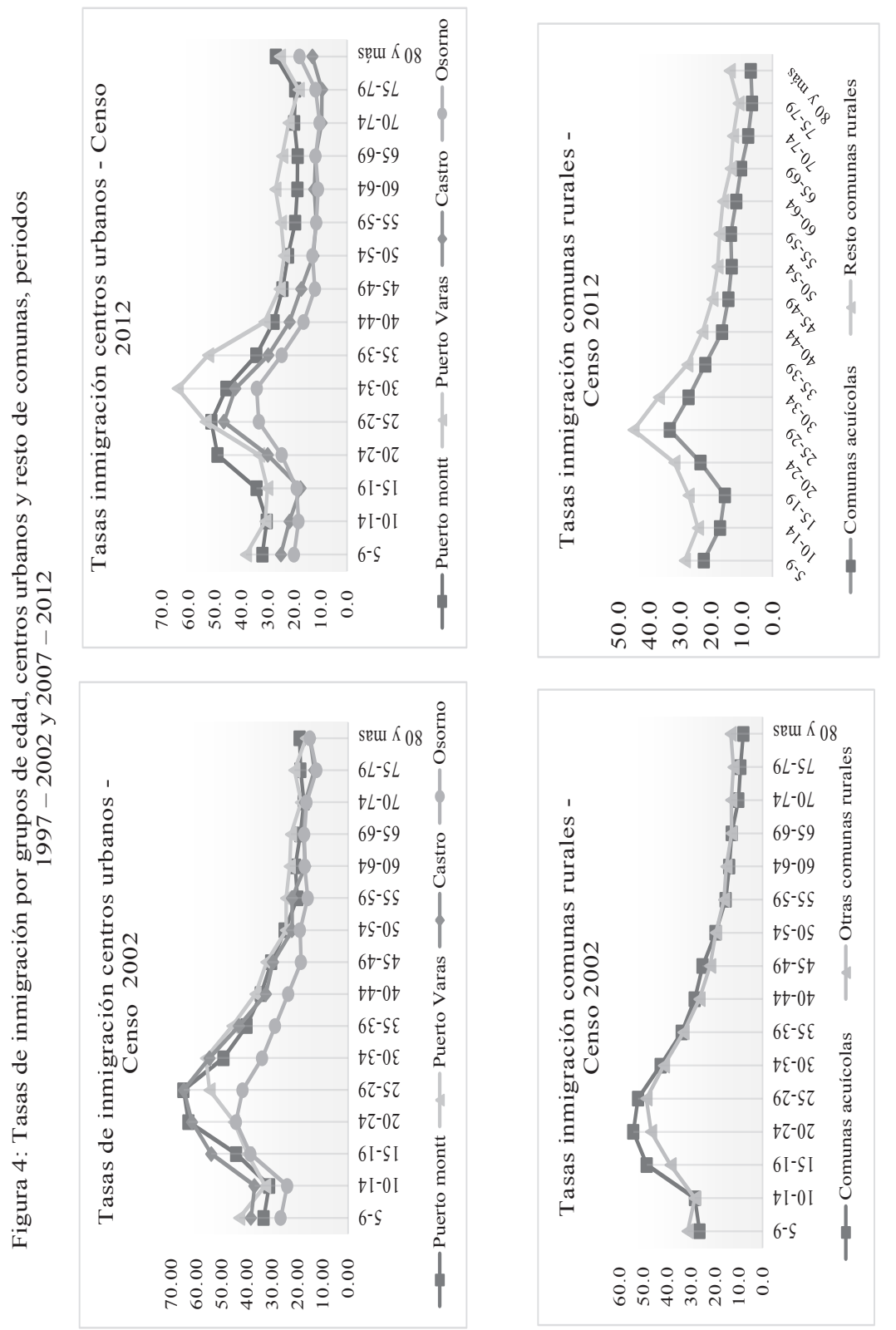

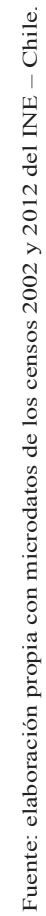


Migración y desarrollo en Chile. Respuestas migratorias a las fases de crecimiento y crisis ... / S. SOTO y F. GIL
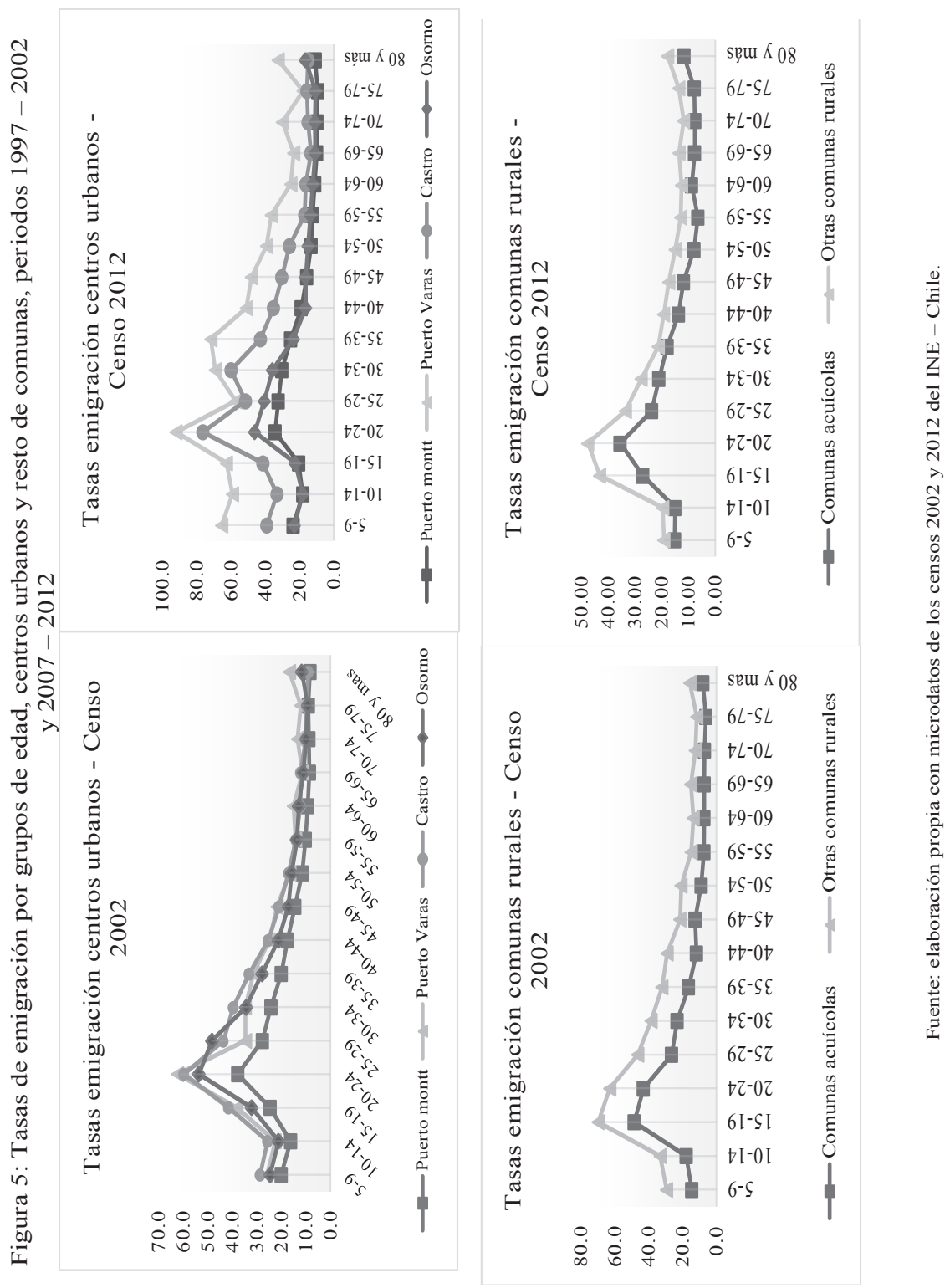


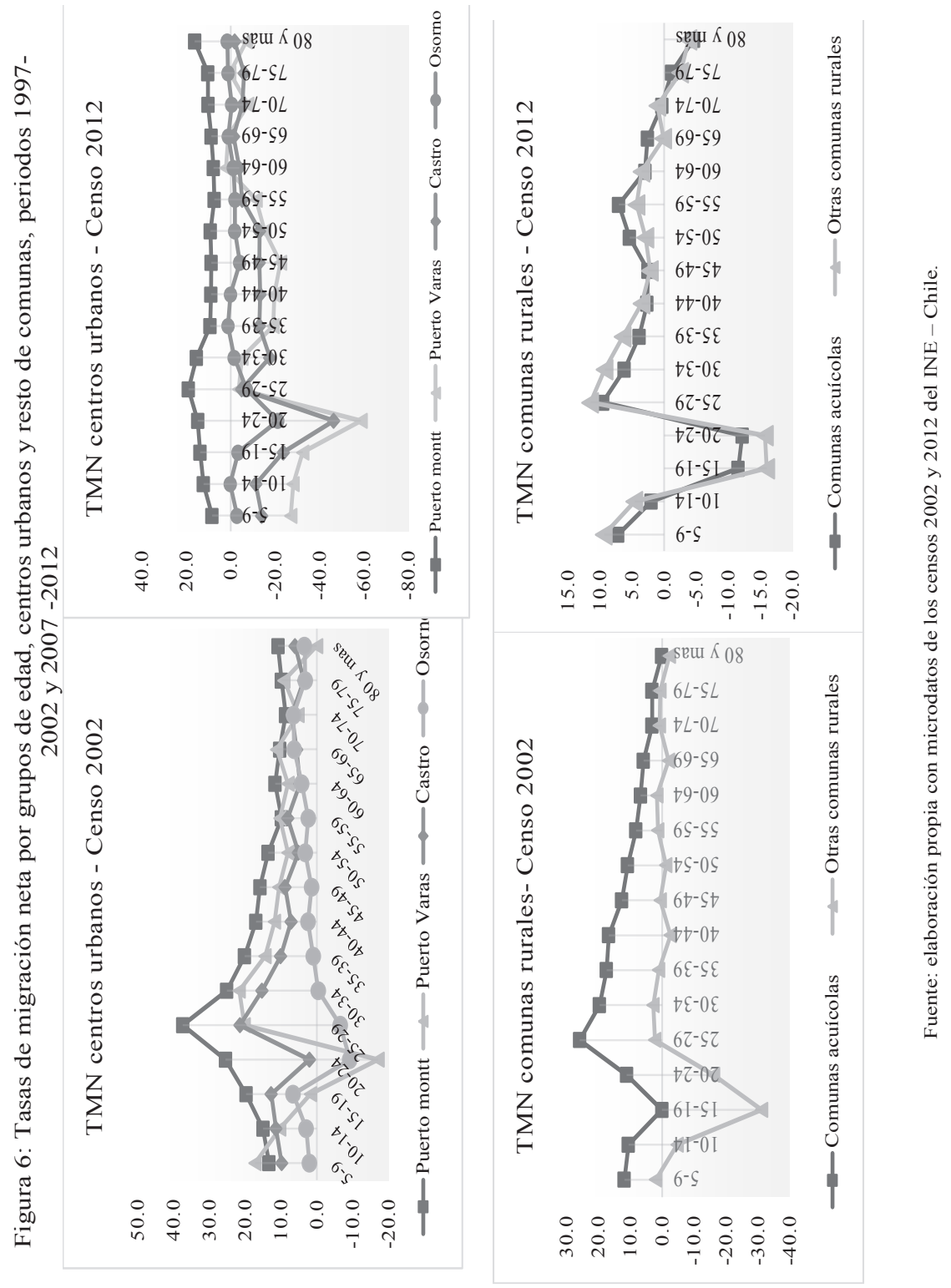


Finalmente, el análisis de las tasas de migración neta (TMN) muestra que Puerto Montt es la única comuna que mantiene una TMN positiva en forma continua y en todas las edades, que además se incrementa entre las edades de 25 a 34 años. Las demás comunas que son centros urbanos tienen tasas negativas en algunos períodes censales y en ciertas edades, especialmente entre los 20 y 24 años, tasas negativas que se han acentuado según los datos del censo de 2012 (Figura 6). Se muestra así, la relevancia econòmica, político y social de la comuna como capital regional.

Las comunas de desarrollo acuícola, que antes de la crisis de 2007-2010 recibía población mayoritariamente entre 25 y 39 años y mantenían sus TMN positivas para todas las edades, han visto modificar sus flujos según los datos del censo 2012 y estas comunas comienzan a perder población a partir de los 15 años y hasta los 24 años. No obstante, pese a la crisis, las comunas acuícolas tienen menores tasas de emigración que las comunas rurales de base agropecuaria.

En general las comunas rurales de la región pierden población a partir de los 15 años y hasta los 24 años aproximadamente, por las razones educativas antes mencionadas. Después de ello las TMN se recuperan y en algunos casos son positivas. Esta es, fundamentalmente, migración de retorno.

Por último, las comunas que se encuentran en condiciones de mayor aislamiento y mala conectividad con los centros urbanos pierden población adulta mayor que emigra a ciudades con más servicios para conseguir una mejor calidad de vida.

\section{ConClusiones Y Discusión}

Tal como se ha señalado en diversos estudios sobre las regiones de Chile (Rodríguez y Espinoza, 2012; Rodríguez y González, 2004; Daher, 2004; Riffo, 2007), en esta investigación es posible observar el impacto que ha tenido el desarrollo de la industria acuícola en el atractivo migratorio de la región. En efecto, los destinos más atractivos de ésta fueron las comunas con mar interior en la provincia de Chiloé, Palena y Llanquihue donde se desarrollan las actividades acuícolas, así como las ciudades capitales de región y provincia. Sin embargo, la fragilidad del desarrollo y atractivo de los territorios que basan su economía en una única actividad queda en evidencia después de la crisis de la industria del salmón, ante la aparición del virus ISA el año 2007. 
Las comunas quedaron desprovistas de fuentes laborales y al no poseer otra actividad productiva se transformaron en territorios de expulsión de población. La capital regional Puerto Montt fue la única excepción.

Las comunas de la región en las que su actividad económica principal es agrícola y forestal fueron sistemáticamente expulsoras de población hasta el censo de 2002; posteriormente, una vez desatada la crisis se modifica esta dinámica y algunas comunas expulsoras comienzan a ganar población. Estos son territorios con un porcentaje de ruralidad sobre 50 por ciento y que tienen una distribución del suelo desigual, existiendo, por una parte, un pequeño grupo de latifundistas que son propietarios de la mayor parte del suelo, y una mayoría de pequeños propietarios con terrenos de uno a cinco hectáreas que tienen una economía de subsistencia. Algunas de estas comunas, sobre todo en la provincia de Osorno, tienen una alta proporción de población Mapuche Huilliche, en las cuales las dinámicas migratorias adquieren características distintas a las demás comunas rurales. La expulsión de población en estas comunas con menores capacidades socioeconómicas se produce a edades tempranas, entre $\operatorname{los} 15$ y 25 años, originada por la consecución de estudios y/o búsqueda de oportunidades laborales.

La situación de rezago y pobreza en estas comunas aumenta con la emigración de la población a temprana edad, quedando desprovista de capital humano y transformándose en una trampa territorial de pobreza (Rodríguez y Busso, 2009). Se verifica entonces que las comunas de la región de Los Lagos son heterogéneas en cuanto al comportamiento de migraciones, y que esta situación se presenta como un hándicap para las comunas con menores capacidades endógenas, pues con la pérdida de población en edad laboral, que generalmente corresponde a quienes tienen más capacidades y mayor nivel educacional, disminuyen las posibilidades de producción en el territorio.

En los periodos analizados, la tendencia de la migración intrarregional muestra que el desarrollo de la industria acuícola acrecentó la heterogeneidad entre las comunas, favoreciendo en cuanto a los intercambios de población a las comunas que se constituyeron como polos de desarrollo, generando "burbujas" al interior de la región. Sin embargo, la dinámica migratoria fue impactada por la crisis económica vivida en la región el año 2007, disminuyendo drásticamente los flujos de población en toda la región y sobre todo en las comunas de desarrollo acuícola, y acortando las diferencias migratorias entre las comunas.

Por otro lado, aparte de estas últimas, existen también comunas que presentan dinámicas migratorias positivas de menor intensidad, por subur- 
banización de la comuna de Puerto Montt. Ello refleja que, si bien predomina la movilidad ligada a las oportunidades laborales en la región de Los Lagos, no se puede desconocer la creciente existencia de movilidad atribuible a factores relacionado con el ciclo de vida, particularmente de parejas que desean tener descendencia o ampliar ésta y que, en consecuencia, cambian de residencia en busca de una mayor calidad de vida (mejores viviendas, entorno medioambiental más adecuado). Ello afecta especialmente a segmentos de población con nivel económico, educativo y cultural medio-altos y sería un fenómeno crecientemente presente en las comunas en torno al lago Llanquihue, particularmente en Puerto Varas, donde se está formando una incipiente conurbación con Puerto Montt, ciudad generadora de estos flujos de suburbanización.

Tomando en consideración lo expuesto en las conclusiones hasta ahora y, de acuerdo a lo que señala De Mattos (2000), es posible explicar las migraciones internas de la región a través del planteamiento de la "continuidad del patrón de estructuración territorial", pues pese a que se identifican polos de actividades productivas en distintas comunas de la región, las gerencias y las operaciones más importantes de las industrias continúan realizándose en algunos centros, como las capitales regionales -en este caso, Puerto Montt—, donde se observa una dinámica migratoria articulada con el resto del país. Por lo demás, aunque la región de los Lagos es una "región commodity" de acuerdo al planteamiento de Daher (1994), no parece haber signos de convergencia al interior de la región como producto de la actividad económica de la industria acuícola, así como tampoco los hay entre las regiones. Por el contrario, los resultados de la investigación muestran que, al menos en términos de atractivo migratorio, las comunas tienden a la divergencia más que a la convergencia durante los periodos de mayor crecimiento económico regional, y únicamente factores coyunturales, como la crisis de salmón, parecen favorecer dinámicas convergentes aunque probablemente de corta duración.

Ante esta situación emerge la importancia del rol de las políticas públicas para regular y contribuir a desencadenar procesos de ajuste territorial, que permitan mitigar de alguna forma la emigración masiva de los territorios más desfavorecidos. En esta línea, las políticas públicas debiesen orientarse, por un lado, a promover el desarrollo local a través de actividades productivas diversificadas de acuerdo a la vocación de los territorios, $\mathrm{y}$ por otro lado, a implementar establecimientos educacionales de enseñanza media técnico-profesional y centros de formación técnica en las comunas con amplias desventajas que reduzcan la fuga de población a edades tem- 
pranas por motivos educacionales y que, al mismo tiempo, puedan funcionar también como un factor de atracción en dichas comunas.

\section{Agradecimientos}

Este trabajo se realizó en el marco del proyecto de $\mathrm{I}+\mathrm{D}+\mathrm{i}$ "Desigualdad social, polarización territorial y formación de espacios vulnerables en las grandes áreas metropolitanas españolas" (CSO2015-65219-C2-1-R), financiado por la Agencia Estatal de Investigación (AEI) y por el Fondo Europeo de Desarrollo Regional (FEDER, UE) y dirigido por los doctores Isabel Pujadas y Fernando Gil Alonso, que además es miembro del grupo de investigación Territori, Població i Ciutadania, reconocido por la Generalitat de Catalunya. Este trabajo fue realizado con el apoyo de CONICYT Chile, Programa Formación de Capital Humano Avanzado, Beca Doctorado en el extranjero convocatoria 2015.

\section{REFERENCIAS BIBLIOGRÁFICAS}

Amtmann, C. A., y Blanco, G., 2001, "Efectos de la salmonicultura en las economías campesinas de la Región de Los Lagos, Chile", en Revista Austral Ciencias Sociales, 5, 93-106.

Chackiel, J., 2009, "Censo, residencia habitual y movilidad territorial”, en Notas de población, Año 36, 88, 21-61.

Daher, A.,1994, "Competencia: regiones ganadores y perdedoras en Chile", en Revista EURE, 20-60, 63-84.

Daher, A., 2004, "Riesgo-país versus riesgo-región: Santiago en el Mercosur", en de Mattos, C., Ducci, M.E., Rodríguez, A. y Yáñez, G. (eds.) Santiago en la Globalización: ¿una nueva ciudad?, Santiago: Ediciones SUR - EURE Libros, 85-114.

De Mattos, C. A., 1995, "Reestructuración, globalización, nuevo poder económico y territorio en el Chile de los noventa", en Revista de Estudios Regionales, 3, 69-100.

De Mattos, C. A., 1999, "Santiago de Chile, globalización y expansión metropolitana: lo que existía sigue existiendo", en Revista EURE, 25-76, 29-56.

De Mattos, C. A., 2000, "Nuevas teorías del crecimiento económico: una lectura desde la perspectiva de los territorios de la periferia", en Revista de Estudios Regionales, 58, 15-40, Instituto de Estudios Urbanos, Pontificia Universidad Católica dc Chile.

Gillet, J. y Olate, C., 2010, La crisis del salmón y el desempleo en la décima región, Tesis de pregrado. Universidad de Chile, Santiago, Chile. 
Massey, D. S., 1990, "Social structure, household strategies, and the cumulative causation of migration", en Population Index, 3-26.

Massey, D. S., Arango, J., Hugo, G., Kouaouci, A., Pellegrino, A. y Taylor, J. E., 1993, "Theories of international migration: A review and appraisal", in Population and development review, 19 (3), 431-466.

Riffo, L., 2007, “Desarrollo económico y Movimiento de Población en Chile”, en Taller nacional sobre migración interna y desarrollo en Chile: diagnóstico, perspectivas y políticas. Santiago: CEPAL, CELADE.

Rodríguez Vignoli, J., 2004, Migración interna en América Latina y el Caribe: estudio regional del período 1980-2000. Serie Población y Desarrollo, 50. Santiago de Chile: CEPAL.

Rodríguez Vignoli, J., 2008, “Distribución espacial, migración interna y desarrollo en América Latina y el Caribe”, en Revista de la CEPAL, 96, 135-155.

Rodríguez Vignoli, J., 2011, "Migración interna en ciudades de América Latina: efectos en la estructura demográfica y la segregación residencial", en Notas de población, Año 37, 93. 135-167.

Rodríguez Vignoli, J. y Espinoza, D., 2012, “Recuperación del atractivo migratorio metropolitano en el período 2004-2009: ¿factores exógenos o endógenos?”, en Revista de Geografia Norte Grande. 51, 95-113.

Rodríguez, J. y Busso, G., 2009, Migración Interna y desarrollo en América Latina entre 1980 y 2005. Santiago de Chile: Libros de la CEPAL, 102.

Rodríguez, J. y González, D., 2004, “Tendencias recientes de la migración interna en Chile: recuperación regional selectiva, desconcentración metropolitana y rururbanización", Documento presentado al Congreso de la Asociación Latino Americana de Población (ALAP), Caxambú.

Rodríguez, J. y González, D., 2006, "Redistribución de la población y migración interna en Chile: continuidad y cambio según los últimos cuatro censos nacionales de población y vivienda", en Revista de Geografia Norte Grande, 35, 7-28.

Todaro, M.P., 1980, "Internal migration in developing countries: a survey", in Population and economic change in developing countries. Chicago: University of Chicago Press. 


\section{RESUMEN CURRICULAR DE LOS AUTORES}

\section{Sylvia Soto Alvarado}

Es Licenciada en Sociología por la Universidad de La Frontera, Chile. Maestra en Planificación Territorial y Gestión Ambiental y ( C ) Doctora en Geografía, Planificación Territorial y Gestión Ambiental por la Universidad de Barcelona. Actualmente es profesora de la escuela de Administración Pública, Universidad Austral de Chile, Sede Puerto Montt. Sus áreas preferentes de estudio son las dinámicas migratorias con especial atención en las transformaciones sociodemográficas, redes sociales e incorporación en la sociedad.

Dirección electrónica: sylviasotoa@gmail.com

Fernando Gil Alonso

Es Licenciado en Geografía e Historia por la Universidad de Barcelona. Postgrado en Métodos y Técnicas para el Estudio de la Población por el Centro de Estudios Demográficos y Doctor en Geografía Humana (rama Demografía) por la Universidad Autónoma de Barcelona. Actualmente es profesor agregado del Departamento de Geografía de la Universidad de Barcelona. Es asimismo miembro del grupo de investigación $\mathrm{Te}$ rritorio, Población y Ciudadanía, dirigido por la Dra. Isabel Pujadas, que ha sido reconocido como grupo de investigación consolidado por la Generalitat de Cataluña. También es co-investigador principal del proyecto $\mathrm{I}+\mathrm{D}+\mathrm{i}$ Desigualdad social, polarización territorial y formación de espacios vulnerables en las grandes áreas metropolitanas españolas (CSO2015-65219-C2-1-R), financiado por la Agencia Española de Investigación (AEI) y por el Fondo Europeo de Desarrollo Regional de la Unión Europea (FEDER, UE). Sus áreas preferentes de estudio son las estructuras y dinámicas demográficas (urbanas, rurales, regionales, nacionales), con especial atención a los flujos migratorios y a la interacción entre dichas migraciones, las estructuras demográficas y el mercado de trabajo. Dirección electrónica: fgil@ub.edu 